\title{
Selective transcatheter arterial infusion of cisplatin in palliative intent for residual bulky tongue cancers after intravenous systemic chemotherapy combined with radiotherapy: Two case reports
}

\author{
Yuki Wada ${ }^{*}$; Aoi Otaka²; Takaharu Miyauchi'; Naoko Fukuiं; Hiromoto Kimura ${ }^{3}$; Manabu Hashimoto ${ }^{1}$ \\ ${ }^{1}$ Department of Radiology, Akita University Graduate School of Medicine, 1-1-1 Hondo, Akita, Akita Japan. \\ ${ }^{2}$ Department of Diagnostic Radiology, Akita Red Cross Hospital, 222-1 Saruta-Nashirozawa, Kamikitate, Akita, Akita Japan. \\ ${ }^{3}$ Department of Otolaryngology, Akita Red Cross Hospital, 222-1 Saruta-Nashirozawa, Kamikitate, Akita, Akita Japan.
}

\section{*Corresponding Authors: Yuki Wada}

Department of Radiology, Akita University Graduate School of Medicine, 1-1-1 Hondo, Akita, Akita, 010-8543, Japan.

Tel: +81-18-834-1111, Fax: +81-18-836-2623;

E-mail: ywada@med.akita-u.ac.jp

Received: Aug 27, 2021

Accepted: Sep 23, 2021

Published: Sep 30, 2021

Archived: www.jcimcr.org

Copyright: (C) Wada Y (2021).

\section{Abstract}

Concurrent radiotherapy with Transcatheter Arterial Infusion (TAI) chemotherapy is reportedly effective in curing locally advanced head and neck cancer. However, the effectiveness and safety of using TAI with palliative or salvage intent after radiotherapy combined with systemic intravenous chemotherapy (IV-CRT) is not well known. Herein, we describe two cases using TAI palliatively for locally advanced residual tongue cancer after IV-CRT, where tumor reduction or tumor progress inhibition could be achieved, thereby improving or maintaining the patients' quality of life without severe toxicities. These cases indicate the effectiveness and safety of using TAI palliatively for locally advanced tongue cancer. Further, we have combined a literature review with the description of these cases.

Keywords: tongue cancer; transcatheter arterial infusion; palliative interventional radiology.

\section{Introduction}

The treatment combining Transcatheter Arterial Infusion (TAI) of cisplatin (CDDP) with Radiotherapy (RT) is known as RADPLAT and is occasionally used as definitive therapy for head and neck cancers $[1,2]$. The important merits of RADPLAT are that it improves the treatment effect due to the increasing density of CDDP in tumor vessels and reduces the side effect of CDDP by using sodium thiosulfate intravenously, which neutralizes CDDP. RADPLAT for locally advanced maxillary and tongue cancer has been mentioned in a Japanese clinical practice guideline for head and neck cancer [3]. However, the superiority of RADPLAT over the combination of systemic intravenous chemotherapy and RT (IV-CRT) has not been established due to the lack of prospective randomized trials and because only a few institutions can perform TAI. Therefore, IV-CRT is widely selected as the first-line treatment for head and neck cancer. When a residual tumor exists after IV-CRT, systemic chemotherapy is selected, unless salvage operations can be applied. However, sufficient systemic chemotherapy is sometimes difficult if the patient condition or laboratory data is not conductive.

We encountered two cases with locally advanced residual tongue cancer after IV-CRT in which palliative TAI could achieve tumor reduction or progress inhibition, thereby improving or maintaining the patients' quality of life without severe toxicities. 
Citation: Wada Y, Otaka A, Miyauchi T, Fukui N, Kimura H. Selective transcatheter arterial infusion of cisplatin in palliative intent for residual bulky tongue cancers after intravenous systemic chemotherapy combined with radiotherapy: Two case reports. J Clin Images Med Case Rep. 2021; 2(5): 1333.

\section{Case series}

\section{Case 1}

An 83-year-old woman presented with spindle cell squamous cell carcinoma of the right edge of the tongue (Figure 1A). Because the locally advanced tumor was inoperable, she had initially been treated with IV-CRT with curative intent using cetuximab for systemic therapy because her renal function was insufficient for using CDDP. Radiotherapy was planned using 4 Megavoltage $X$-rays with opposing portal irradiation from bilateral sites up to $70 \mathrm{~Gy}$ in 35 fractions (Figure 1B). However, it was stopped at 50 Gy following severe radiation dermati-

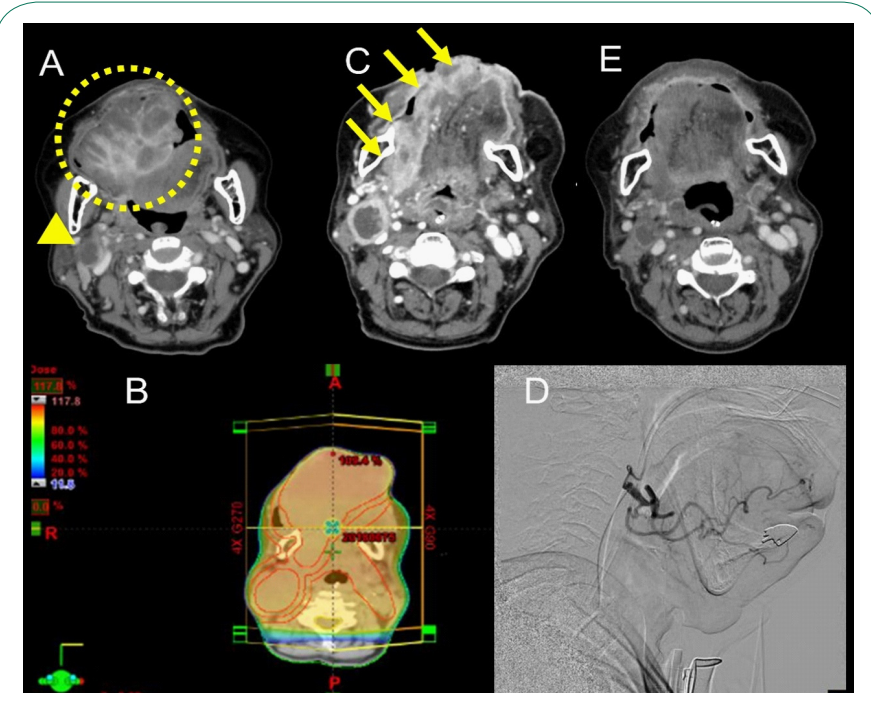

Figure 1: (1A): Computed Tomography (CT) image before the initiation of intravenous systemic chemotherapy combined with radiotherapy (IV-CRT), shows a huge tumor originating from the right edge of the tongue and occupying the oral cavity (circle). In addition to the necrotizing enlargement of the right internal deep cervical lymph node (arrowhead), numerous enlarged lymph nodes were found in the neck bilaterally (not shown).

(1B): Shows the dose distribution of the initial radiotherapy planning at the level of the primary tongue mass. Bilaterally opposed portal irradiation with 4 Megavoltage X-rays was used. After $42 \mathrm{~Gy}$, the irradiation field was shrunk to avoid over-exposure of the spinal cord (not shown).

(1C): The CT image after IV-CRT, shows the partial response of the primary lesion; the bulky mass still remains (arrows).

(1D): Shows the angiography of the common trunk of the right lingual artery and right facial artery. Angiography was performed from the right femoral artery using the Seldinger technique with a blood access sheath (Medikit Supersheath', 4 Fr. $25 \mathrm{~cm}$, Medikit, Tokyo, Japan). A guiding catheter (Medikit's Angiographic Catheter VTA type, 4 Fr. $100 \mathrm{~cm}$, Medikit, Tokyo, Japan) was placed at the right external carotid artery induced with guiding wire (Radifocus ${ }^{\circ}$ Guidewire E-type, Terumo, Tokyo, Japan), and the common trunk of the right facial artery and lingual artery was selected with a microcatheter (Carnelian PIXIE', 1.7 Fr. 135 cm, Tokai Medical Products Inc., Aichi, Japan) and micro guidewire (Transend ${ }^{\mathrm{TM}}$ Guidewires, 0.014 inches $182 \mathrm{~cm}$, Boston Scientific, MA, USA). The pre-transcatheter arterial infusion (TAI) CT scan showed that the main feeder of a huge tongue tumor was the common trunk of these arteries. Therefore, we infused $50 \mathrm{mg}$ of cisplatin from here twice, with a three-week interval.

(1E): The CT image after TAI, reveals sufficient primary tumor reduction, after which the patient was able to close her mouth. tis and mucositis of the oral cavity. IV-CRT could achieve only a partial local response; the residual local tumor was still too large for the patient to be able to close her mouth (Figure 1C). Additionally, the appearance of multiple lung metastases was observed on Computed Tomography (CT) scans the next day, after completing IV-CRT. Because nivolumab, which is used for platinum-resistant head and neck cancer, was not covered by the Japanese insurance system at the time, S-1 (tegafur, gimeracil, oteracil) was administered as second-line chemotherapy for the patient. However, no treatment effect was observed and S-1 was stopped after only two weeks. No chemotherapy regimens were expected to be effective and tolerable for her physical condition, however, to improve her chief complaint of thirst, which was caused by an inability to close her mouth, we planned TAI of CDDP one month after finishing IV-CRT in order to treat the local tongue mass. Pre-TAI CT images and 3D-CT angiography showed that the local lesion was fed by the right common trunk of the lingual artery and facial artery. We injected $50 \mathrm{mg}$ of CDDP from the trunk via the transcatheter twice with an interval of three-weeks (Figure 1D). After TAI, the local lesion reduced significantly (Figure $1 \mathrm{E}$ ) and she could close her mouth satisfactorily. The patient's renal function before and after TAl could be maintained with hydration. However, the multiple lung metastases progressed after the second TAI and she died one month after the second TAI. The local tumor was controlled until death, and mouth could closure was possible.

\section{Case 2}

A 63-year-old man presented with squamous cell carcinoma of the right base of the tongue, crossing the midline, and invading the left side. Because the locally advanced tumor was inoperable, the patient had initially been treated with IV-CRT in curative intent using cetuximab, as his renal function was insufficient for CDDP. Radiotherapy was planned using 4 megavoltage $X$-rays with opposing portal irradiation from bilateral sites up to $66.6 \mathrm{~Gy}$ in 37 fractions (Figure 2B). The CT image two weeks after finishing CRT revealed a partial response of the primary tongue tumor and lymph node metastases to IVCRT (Figure 2C). However, the mass volume was still large and considered to worsen his quality of life. We planned the TAI of CDDP palliatively in order to maintaining or improving his quality of life by reducing tumor volume with the therapy. Pre-TAI CT images and 3D-CT angiography showed that the residual lesion straddled bilaterally and was fed by the bilateral lingual arteries. Although injections into the bilateral arteries likely provide better local control than injections into the unilateral artery, they increase the treatment time and patient burden. Finally, we decided on the TAI of $80 \mathrm{mg}$ CDDP into only the main right lingual artery because the therapy was intended to be palliative; treatment of the entire tumor was not mandatory and reducing the burden of treatment on the patient was important. (Figure 2D). The procedure was performed one month after IV-CRT. After TAI, the size of the tumor on the left side of the tongue not injected with CDDP had increased. However, the enhancement effect within the right tongue artery feeding area was reduced and the tumor size was stabilized; this was thought to be a treatment effect (Figure 2E). Because of the progression of the tumor on the right side, which was inhibited but not clearly reduced in size, and the progression of the tumor in the 
area where CDDP was not injected, the patient was switched to systemic chemotherapy with S-1 instead of local treatment with TAl. He was found dead in his home two months after the TAI. The cause of death was not determined, however, the examination three weeks prior to death had confirmed that the primary tongue tumor had not increased in size.

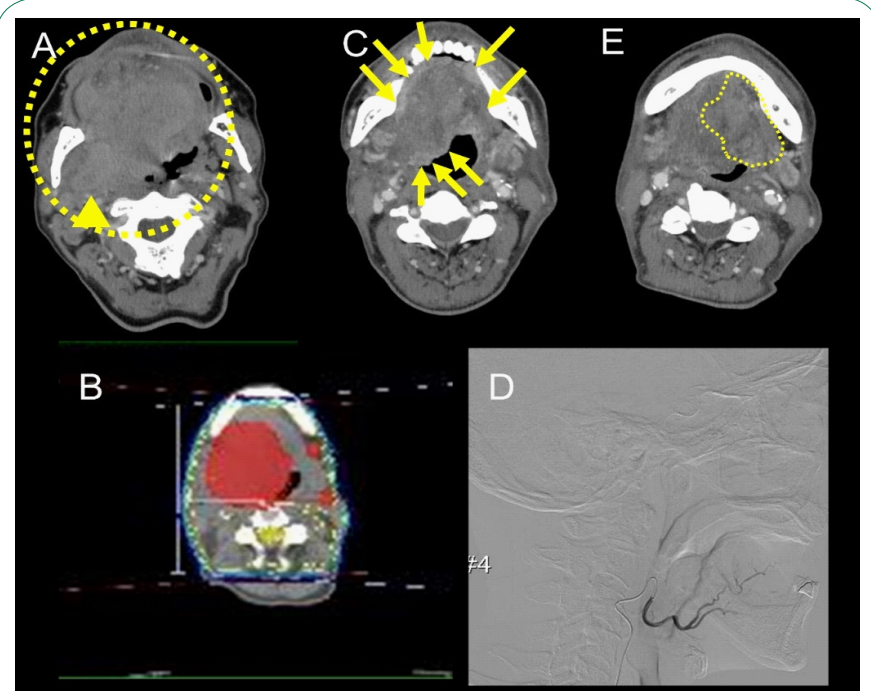

Figure 2: (2A): Computed Tomography (CT) image before the initiation of intravenous systemic chemotherapy combined with radiotherapy (IV-CRT) shows that the huge tongue tumor occupying the oral cavity originated from the tongue base on the right side and crossed the midline to invade the left half of the tongue (circle). The right submandibular lymph node is swollen along with the primary tumor (arrowhead), and the right internal deep cervical lymph node swelling can also be seen. Many other enlarged lymph nodes were also present on both sides of the neck (not shown).

(2B): Shows the dose distribution of radiotherapy at the level of the primary tongue mass (The image is pixelated due to the quality of the radiation therapy planning system). Bilaterally opposed portal irradiation with 4 Megavoltage X-rays was used. After 41.4 Gy, the irradiation field was shrunk to avoid over-exposure of the spinal cord (not shown).

(2C): The CT image after IV-CRT shows the partial response of the primary lesion. However, the bulky tumor, enhanced with contrast medium, can still be detected along the edge bilaterally and along the base of the tongue on the right (arrows).

(2D): Shows the angiography of the right lingual artery. Angiography was performed from the right femoral artery using the Seldinger technique with a blood access sheath (Medikit Supersheath", 4 Fr. $25 \mathrm{~cm}$, Medikit, Tokyo, Japan). A guiding catheter (Medikit's Angiographic Catheter VTA type, 4 Fr. 100 cm, Medikit, Tokyo, Japan) was placed at the right common carotid artery induced with guiding wire (Radifocus ${ }^{\circ}$ GuidewireM E-type, Terumo, Tokyo, Japan), and the common trunk of the right facial artery and lingual artery was selected with the microcatheter (Carnelian PIXIE', 1.7 Fr. $135 \mathrm{~cm}$, Tokai Medical Products Inc., Aichi, Japan) and micro guidewire (Transend ${ }^{\mathrm{TM}}$ Guidewires, 0.014 inches $182 \mathrm{~cm}$, Boston Scientific, MA, USA). The Pre-Transcatheter Arterial Infusion (TAI) CT scan showed that the feeders of the huge tongue tumor were the bilateral lingual arteries, and the right one was related to the primary tumor more than the left one. Therefore, we infused 80 $\mathrm{mg}$ of cisplatin from the right lingual artery using sodium intravenous thiosulfate, which neutralizes CDDP.

(2E): The CT image after TAl shows tumor progression on the left side of the tongue where CDDP had not been injected (yellow dotted line area). However, a reduction in the enhanced effect was observed at the right edge and the right base of the tumor, and the tumor size of the injected area was stable; this was thought to be a treatment effect of TAl.

\section{Discussion}

Although palliative intent TAI after IV-CRT has been inadequately investigated, several studies on the effectiveness of TAI as first-line monotherapy or combined therapy with radiation or surgery are available. A phase I study to evaluate the tolerated dose of CDDP in TAI revealed that the overall response (complete or partial response) and complete response rates were $86 \%$ and $41 \%$ for untreated stage III/IV head and neck cancer, and $62 \%$ and $25 \%$ for locally recurrent head and neck cancer, respectively [4]. Another study on TAl as neoadjuvant therapy before surgery or radiotherapy for stage III/IV squamous cell carcinoma of the mouth showed that the overall and complete response rates were $95 \%$ and $71 \%$, respectively [5]. The response rate of nivolumab, which is used for recurrent of residual head and neck cancer after IV-CRT, is reported to be $13.3 \%$ in phase III randomized trial [6]. Although each patient's characteristics in these studies were different, the reported objective response rate of TAl for head and neck cancer was not found to be inferior to that of nivolumab.

Another advantage of TAI is that it shows fewer toxicities than does systemic chemotherapy. One report showed reversible grade $1 / 2$ toxicities in $14.8 \%$ of the patients and grade $3 / 4$ toxicities in only $1.1 \%$ of the patients [4]. One of the major complications in the systemic use of CDDP is renal impairment, however, previous study showed grade 2 or worse renal impairment has been shown in only $1 \%$ of the TAI-CRT [7].

For providing effective TAI, it is important to evaluate and select the feeding arteries and modify the chemotherapy doses for each feeder. A previous study that evaluated the feeding arteries of 20 primary tongue cancers treated with TAl showed that the main feeding artery was the ipsilateral lingual artery in $75 \%$ of the cases and the contralateral lingual artery in $25 \%$ [8]. They also showed that $50 \%$ of all tumors were fed by only one artery, while the other $50 \%$ were fed by multiple arteries. Additionally, they revealed that a tumor size $>4 \mathrm{~cm}$, extension to the contralateral side, and involvement of the extrinsic muscles of the tongue were risk factors for multiple feeding arteries [8]. However, the necessity of infusing into all feeding arteries or appropriateness of infusing only into the main feeding artery has not been investigated enough. One report suggested that even if the tumor crosses the midline, it is nourished mainly from the side where the tumor originates and the contralateral side is less involved; however, bilateral treatment should be considered if it crosses more than $1 \mathrm{~cm}$ beyond the midline [9]. Another report showed that tumor response was better with a unilateral injection than with a bilateral injection, when the total amount of the anticancer drug was the same [10]. As infusing into multiple feeding arteries prolongs the procedure compared to infusing into a single feeding artery, it is preferable to infuse into a single feeding artery and repeat the treatment as needed, especially in patients whose condition is too poor to be eligible for sufficient systemic chemotherapy, as the present case 2 .

\section{Conclusion}

In conclusion, our cases suggest that the palliative TAI of CDDP for residual head and neck cancer after IV-CRT is an effective and safe treatment choice. This therapy can be potentially utilized for patients considered for the best supportive care instead of further systemic chemotherapy due to poor conditions or laboratory data. 


\section{References}

1. Homma A, Oridate N, Suzuki F, Taki S, Asano T, et al. Superselective high-dose cisplatin infusion with concomitant radiotherapy in patients with advanced cancer of the nasal cavity and paranasal sinuses: A single institution experience. Cancer. 2009; 115: 4705-4714.

2. Kano S, Homma A, Oridate N, Suzuki F, Hatakeyama $\mathrm{H}$, et al. Superselective arterial cisplatin infusion with concomitant radiation therapy for base of tongue cancer. Oral Oncol. 2011; 47: 665-670.

3. Nibu KI, Hayashi R, Asakage T, Ojiri H, Kimata Y, et al. Japanese Clinical Practice Guideline for Head and Neck Cancer. Auris Nasus Larynx. 2017; 44: 375-380.

4. Robbins KT, Storniolo AM, Kerber C, Vicario D, Seagren S, et al. Phase I study of highly selective supradose cisplatin infusions for advanced head and neck cancer. J Clin Oncol. 1994; 12: 21132120.

5. Hirai T, Korogi $Y$, Hamatake S, Nishimura R, Baba Y, et al. Stages III and IV squamous cell carcinoma of the mouth: Three-year experience with superselective intraarterial chemotherapy using cisplatin prior to definitive treatment. Cardiovasc Intervent Radiol. 1999; 22: 201-205.
6. Ferris RL, Blumenschein G Jr, Fayette J, Guigay J, Colevas AD, et al. Nivolumab for recurrent squamous-cell carcinoma of the head and neck. N Engl J Med. 2016; 375: 1856-1867.

7. Rasch CRN, Hauptmann M, Schornagel J, Wijers O, Buter J, et al. Intra-arterial versus intravenous chemoradiation for advanced head and neck cancer: Results of a randomized phase 3 trial. Cancer. 2010; 116: 2159-2165.

8. Kamitani T, Kawanami S, Asayama Y, Matsuo Y, Yonezawa M, et al. Feeding arteries of primary tongue cancers on intra-arterial infusion chemotherapy. Cardiovasc Intervent Radiol. 2016; 39: 227-232.

9. Robbins KT, Howell SB, Williams JS. Intra-arterial chemotherapy for head and neck cancer: Is there a verdict? Cancer. 2010; 116: 2068-2070.

10. van den Broek GB, Rasch CRN, Pameijer FA, Peter E, van den Brekel MWM, et al. Pretreatment probability model for predicting outcome after intraarterial chemoradiation for advanced head and neck carcinoma. Cancer. 2004; 101: 1809-1817. 